\title{
Aberrant Epigenetic Modifications in Male Infertility ${ }^{\dagger}$
}

\author{
Singh Rajender ${ }^{*}, 1$ and Ashok Agarwal ${ }^{2}$ \\ ${ }^{I}$ Central Drug Research Institute, Lucknow, U.P., India \\ ${ }^{2}$ Centre for Reproductive Medicine, Cleveland Clinic, Cleveland, Ohio, USA
}

\begin{abstract}
Epigenetic modifications change gene expression in such a way that gene action is affected without change in DNA sequence. Several genes playing crucial roles in spermatogenesis are known to be epigenetically regulated. Epigenetic modifications affect spermatogenesis, and aberrant epigenetic modifications at certain loci are linked to male infertility. Epigenetic modifications could be the mechanism by which several environmental hazards such as exposure to endocrine disruptors affect spermatogenesis. Imprinting as an effect of changes in the epigenome has the potential to change gene expression up to several generations leading to inheritance of the modified phenotype. In the present review, we have gathered evidence that epigenetic modifications affect spermatogenesis and contribute to male infertility.
\end{abstract}

Keywords: Epigenetic abberations, spermatogenesis, male infertility, methylation.

\section{INTRODUCTION}

Among various causes of male infertility, genetic factors have explained $10-15 \%$ of cases, with Y-deletions being most commonly observed abnormality [1]. The emphasis of male infertility etiology has recently shifted to epigenetic modifications. The genome is defined as the total amount of information encoded by the nucleotide sequence of an organism's DNA. The epigenome is the modifications made in gene expression by changing DNA and histone structure without altering the DNA sequence itself. Epigenetic modifications include DNA methylation, posttranslational histone modifications and chromatin remodeling. These changes can have short or long-term effects and could be trans-generational [2]. DNA is bound to histone proteins which determine the compactness of DNA. Gene expression in a particular region of DNA is determined by core histones in a way that tightly packed regions named as heterochromatin are transcriptionally inactive; conversely, regions that are bound loosely to histones are called euchromatin and are transcriptionally active. Epigenetic modifications play important role in determining the compactness of DNA in a particular region. Therefore epigenetic changes could be synonymous with changes in gene expression. While the genetic code is considered to be static, or the same in every cell for an organism's entire life, the epigenetic code is dynamic and tissue-specific [3]. Therefore, genetic code defines permanent imprint of information determining the phenotype and characteristics, while epigenetic code provides dynamic imprint to finely tune the phenotype and characteristics according to environmental and other factors. The possibility of reversal of epigenetic changes due to dynamism of the process forms the basis of therapy based on epigenenetics [4]. The present review provides an update on

\footnotetext{
*Address correspondence to this author at the Division of Endocrinology, Central Drug Research Institute, Lucknow, U.P., 226001 India; Tel: +91522-2613894, Ext. 4395; Fax: +91-522-2623405; E-mail: rajender_singh@cdri.res.in
}

${ }^{\dagger}$ CDRI communication number for this article is 8054 evidence that disturbed epigenetic changes may be associated with male infertility.

\section{OVERVIEW OF EPIGENETIC PROCESSES}

Of the known epigenetic processes, the three most common and best characterized are DNA methylation, histone modification and chromatin remodeling. These processes though involve different mechanisms to modify gene expression, have same end effect. The processes are briefly summarized below.

\section{DNA Methylation}

DNA methylation particularly at the $\mathrm{CpG}$ dinucleotides within promoter regions of genes conveys important epigenetic information about gene expression. These dinucleotides consist of cytosine bound to guanine by a phosphodiester bond. $\mathrm{CpG}$ dinucleotides can be found in clusters that have been termed $\mathrm{CpG}$ islands. Takai and Jones defined a $\mathrm{CpG}$ island as a 500-base pair stretch of DNA with a $\mathrm{C}+\mathrm{G}$ content of $55 \%$ or greater and a $\mathrm{CpG}$ frequency of at least 0.65 of the statistically expected value [5]. CpG islands have been found near promoters [6], indicating that they play a role in regulating gene expression. Indeed, hypermethylation of DNA in CpG islands is associated with the maintenance of gene suppression, while hypomethylation in these regions is associated with gene expression [7]. Hypermethylation is also found in centromeric and pericentric heterochromatic sites [8]. In vertebrates, $\mathrm{CpG}$ islands are often associated with "housekeeping" genes, which are required for general cell functions, as well as certain tissue-specific genes [9]. Three DNA methyltransferases, DNMT1, DNMT3A and DNMT3B have been identified as DNA methylation enzymes in eukaryotic cells $[10,11]$. DNMT3A and DNMT3B are considered to be responsible for de novo methylation and are important in the dynamic process of DNA methylation during embryogenesis and pathogenesis $[12,13]$. DNMT1 is the enzyme responsible for maintaining the already established DNA methylation pattern $[14,15]$. 
Whereas the process of DNA methylation has been well characterized, DNA demethylation remains less well characterized [16].

\section{Histone Modifications}

Histones pack DNA compactly in the cell and determine availability of particular DNA sequences for gene expression. The N-termini of histone tails contain amino acid residues that are affected by methylation, acetylation, phosphorylation, ubiquitylation and sumoylation. The sum of these modifications and the information they communicate is referred to as the histone code. Methylation is one of the most prevalent histone post-translational modifications. It is monitored by histone methyltransferases (HMTases) and is generally associated with gene silencing. Methylation of $\mathrm{H} 3 \mathrm{~K} 9$, for example, is a classic indication of gene silencing and is commonly found in heterochromatin, as well as silenced promoters [17]. In some cases, however, methylation of arginine and lysine residues can lead to gene activation [18]. For example, methylation of histone H3K4 is implicated in gene expression [19]. More than one methyl group may be transferred to a single amino acid residue. The location and number of methyl groups in a region of DNA convey a specific epigenetic signal.

Histone acetylation is associated with increased levels of transcription and is modulated by both histone acetyl transferases (HATs) and histone deacetylases (HDACs). HATs activate gene expression, while HDACs inhibit gene expression [20]. Acetylated lysines are specifically recognized by bromodomain-containing proteins and act to enhance chromatin remodeling [21]. Phosphorylation of histones occurs on serine residues and generally leads to gene activation [20]. However, chromosome condensation and gene silencing is seen when the histone variant H2AX is phosphorylated [22]. Covalent modification of histones by ubiquitylation of lysine residues can cause both gene expression and repression. For example, addition of ubiquitin to histone $\mathrm{H} 2 \mathrm{~A}$ is linked to gene silencing [23] whereas ubiquitylation of H2B is linked to gene activation [24]. Lysines may also undergo sumoylation, or the attachment of small ubiquitin-related modifier proteins [SUMOs] [25]. This not only serves to silence gene expression, but also inhibits other types of histone modifications like acetylation [25].

\section{Chromatin Remodeling}

In contrast to the two previously described epigenetic processes, chromatin remodeling is not based on covalent interactions. Instead, ATP-dependent chromatin remodeling complexes use energy from hydrolyzing ATP to alter the location and/or structure of nucleosomes [26]. This can change the available of genes for transcription and can lead to either gene expression or silencing [26]. In yeast, the positions of nucleosomes can generally be predicted based on the primary DNA sequence; this is because nucleosomes must be situated in regions where the DNA can bend [27]. Chromatin remodeling complexes primarily act by sliding histones along the DNA, but they can also twist, spool and bulge the DNA [28]. Two of the common families of such complexes include the SWI/SNF family and ISWI family [29]. The SWI/SNF and ISWI families are ubiquitous, as well as they act in a non-specific fashion on various loci [30]. Each family has a conserved catalytic ATPase subunit to carry out its function [29].

\section{EPIGENETIC ABERRATIONS IN MALE INFER- TILITY}

There is enough evidence to support that at least some spermatogenic genes are epigenetically regulated. A recent spurt in the number of studies analyzing epigenetic aberrations in male infertility is seen. Eventually, epigenetic modifications are being considered to be very important for normal fertility. Endocrine disruptors have been proposed to affect fertility by epigenetic alterations, though only few of these have been subjected to scientific studies; vinclozolin being the most well studied. Only limited data is available on epigenetic concerns in assisted reproduction but concern has been raised about the possible differences in the children conceived using in vitro and in vivo methods as a result if differential modification.

\section{ENDOCRINE DISRUPTORS, EPIGENETIC MODIFI- CATIONS AND MALE INFERTILITY}

Endocrine disruptors induced epigenetic changes may have wide implication in disease etiology [31] and evolution [32]. In particular exposure during embryonic gonadal development and sex-determination are capable of inducing adult onset disease that may perpetuate across generations $[31,33]$. Exposure to vinclozolin, a fungicide commonly used in agriculture and known to be an anti-androgenic endocrine disrupting compound, provides a classical example in this field [34]. Exposure to vinclozolin results in spermatogenic cell defect in the testis of the coming generations [33, 35]. Additional transgenerational diseases develop as animals age (6-14 months), including increased frequencies of tumors, prostate disease, kidney disease, immune abnormalities and other defects in spermatogenesis [36]. Vinclozolin-induced transgenerational effects have been correlated with DNA methylation [33]. The effect of endocrine disruptors on epigenome is transduced during germ line migration, between embryonic days 8 to 14 . The comparable period in the human is between 6 weeks and mid gestation. Germ line during this developmental period is undergoing major reprogramming in its global DNA methylation status [37]. Demethylation to erase the parental epigenetic modifications is followed by re-methylation to establish new sex specific epigenetic pattern.

Looking at the trans-generational effects, GuerreroBosagna et al., (2010) conducted a study to investigate genome-wide promoter DNA methylation alterations in the sperm of F3 generation rats whose F0 generation mother was exposed to vinclozolin [38]. The study used analysis on 52 different regions with statistically significant altered methylation in the sperm promoter epigenome. Mass spectrometry bisulfite analysis was used to map the CpG DNA methylation and confirm 16 differential DNA methylation regions, while the remainder could not be analyzed due to bisulfite technical limitations. Analysis of these validated regions identified a consensus DNA sequence (motif) that is associated with $75 \%$ of the promoters. Interestingly, only $16.8 \%$ of a random set of 125 promoters contained this 
motif. One candidate promoter (Fam111a) was found to be due to a copy number variation $(\mathrm{CNV})$ and not a methylation change, suggesting that initial alterations in the germline epigenome may promote genetic abnormalities such as induced $\mathrm{CNV}$ in later generations. This study identified differential DNA methylation sites in promoter regions three generations after the initial exposure and identifies common genome features present in these regions. In addition to primary epimutations, a potential indirect genetic abnormality was identified, and both are postulated to be involved in the epigenetic transgenerational inheritance observed. This study confirms that an environmental agent has the ability to induce epigenetic transgenerational changes in the sperm epigenome [38].

\section{CHROMATIN REMODELLING AND MALE INFER- TILITY}

Histone modifications regulate gene expression after fertilization by deciding compactness of a region of DNA. Histone modifications are responsible for chromatin remodeling and determination of the availability of a particular region of DNA for transcription. Steilmann et al., 2010 analyzed the interaction between the bromodomain testisspecific (BRDT) gene and differentially modified histones in human spermatozoa [39]. The BRDT transcript level was studied to identify possible correlations between epigenetic changes, mRNA level and subfertility associated with impaired sperm chromatin condensation. Interestingly, reduced binding of investigated modified histone modifications was observed in the BRDT promoter of subfertile patients. Different mRNA levels of BRDT have been detected in a group of infertile patients, as well as in fertile men. Enrichment of methylated histones within the BRDT promoter of fertile sperm suggests that this epigenetic mark may cause repression of BRDT after fertilization, and may be changed in infertile patients. The authors suggested that reduced histone methylation in the promoter of BRDT may be associated with increased transcript levels in subfertile patients [39].

Chromatin remodeling in human spermatogenesis occurs during the replacement of histones with transition proteins followed by protamines in spermatids from Step 3 to Step 5 [reviewed in 40]. This exchange is supported by the presence of acetylated histones [41] and chromatin remodelling factors [42]. To date, 10 chromatin remodelling factor protein families are known: SWI/SNF complex components, polycomb-group genes, chromobox/heterochromatin protein 1 (HP1) homologues, bromodomain proteins, chromodomain/ helicase/DNA-binding domain (CHD) proteins, nucleosome remodelling and histone deacetylase (NuRD) complex components, plant homeodomain (PHD) proteins, inhibitor of growth (ING) family members, methyl-CpG DNA-binding domain (MBD) proteins and the CCCTC-binding factor (zinc finger protein) [43].

Steilmann et al., (2010) analyzed whether a different chromatin remodelling factor expression pattern exists between normal spermatogenesis and round spermatid maturation arrest as potential reason for impaired spermatogenesis and idiopathic male infertility [43]. The expression pattern ranges from a few high expressed genes in round spermatid maturation arrest to a multitude of genes (74) which are more highly expressed in normal spermatogenesis than in maturation arrest. A total of 22 genes showed a significant difference between normal spermatogenesis and round spermatid maturation arrest (1 gene was up-regulated and 21 genes were down-regulated in the developmental arrest). The significantly different expression of chromatin remodelling factors between normal spermatogenesis and round spermatid maturation arrest may lead to impaired epigenetic information and aberrant transcription during sperm development representing one possible reason for developmental arrest of round spermatids [43].

\section{DNA METHYLATION AND MALE INFERTILITY}

Methylenetetrahydrofolate reductase (MTHFR) is a key regulatory enzyme involved in folate metabolism, DNA synthesis and remethylation reactions. MTHFR catalyses the reduction of 5,10-methylenetetrahydrofolate to 5-methyltetrahydrofolate, which is the methyl donor for remethylation of homocysteine to methionine. Methionine is in turn converted to S-adenosylmethionine, a methyl donor used in many reactions whereby substrates such as DNA, RNA, hormones and lipids are methylated. It has been shown that MTHFR deficiency, in addition to folate deficiency, hinders the methylation of a wide variety of substrates, including proteins, DNA, RNA, and histones because of decreased methionine supply $[44,45]$. Since abnormal DNA methylation of imprinted genes has been shown to be associated with oligozoospermia [46], spermatogenesis may be particularly vulnerable to changes in the methyl pool brought about by deficiency in MTHFR.

Khazamipour et al., (2009) compared the methylation status of the promoter region of MTHFR in male patients with non-obstructive azoospermia (NOA) and obstructive azoospermia without anomalies of spermatogenesis [47]. In peripheral blood samples, no differences in the methylation profile of the promoter region of MTHFR were observed between patients and controls. In testis biopsies, hypermethylation was detected in $53 \%$ of the patients with NOA compared with $0 \%$ of patients with obstructive azoospermia $(\mathrm{P}=0.03)$. The authors concluded that hyper-methylation in testis DNA from NOA patients is specific and not due a general methylation defect, and suggested that epigenetic silencing of MTHFR could play a role in azoospermic infertility [47].

In another study, Hammoud et al., (2010) examined CpG methylation patterns in infertile and fertile donors at seven imprinted loci sequenced: LIT1, MEST, SNRPN, PLAGL1, PEG3, H19, and IGF2 [48]. At six of the seven imprinted genes, the overall DNA methylation patterns at their respective differentially methylated regions were significantly altered in both infertile patient populations. When comparing the severity of methylation alterations among infertile patients, the oligozoospermic patients were significantly affected at mesoderm-specific transcript (MEST), whereas abnormal protamine patients were affected at KCNQ1, overlapping transcript 1 (LIT1), and at small nuclear ribonucleoprotein polypeptide $\mathrm{N}$ (SNRPN). The authors concluded that patients with male factor infertility had significantly increased methylation alteration at six of seven 
imprinted loci tested, with differences in significance observed between oligozoospermic and abnormal protamine patients [48].

Jmjd1a is a key epigenetic regulator expressed in the testis. It specifically demethylates mono- and di-methylated histone $\mathrm{H} 3$ lysine 9 (H3K9me1 and H3K9me2) but not trimethylated $\mathrm{H} 3 \mathrm{~K} 9$ ( $\mathrm{H} 3 \mathrm{~K} 9 \mathrm{me} 3)$. This gene is expressed in pachytene and secondary spermatocytes [49]. Disruption of the Jmjd1a gene in mice significantly increased H3K9me1 and $\mathrm{H} 3 \mathrm{~K} 9 \mathrm{me} 2$ levels in pachytene spermatocytes and early elongating spermatids without affecting H3K9me3 levels. Concurrently, the levels of histone acetylation were decreased in Jmjd1a knock-out germ cells. This suggests Jmjdla promotes transcriptional activation by lowering histone methylation and increasing histone acetylation. Interestingly, the altered histone modifications in Jmjd1adeficient germ cells caused diminished cAMP-response element modulator (Crem) recruitment to chromatin and decreased expression of the Crem coactivator Act and their target genes Tnp1 (transition protein 1), Tnp2, Prm1 (protamine 1), and Prm2, all of which are essential for chromatin condensation in spermatids. In agreement with these findings, Jmjd1a deficiency caused extensive germ cell apoptosis and blocked spermatid elongation, resulting in severe oligozoospermia, small testes, and infertility in male mice. These results indicate that the Jmjdla-controlled epigenetic histone modifications are crucial for Cremregulated gene expression and spermatogenesis [49].

The DAZ gene family of the Y-chromosome plays key roles in germline establishment and gametogenesis, with its members encoding for germ cell-specific RNA binding proteins involved in transcript transport/storage, translation initiation and protein regulation. Fittingly, the full deletion of the DAZ copies, as observed in complete AZFc deletions, results in a hypospermatogenesis phenotype [50], whereas studies in mammalian models have demonstrated that DAZL deficiencies lead to spermatogenic arrest [51]. Owing to their extensive similarities, DAZ and DAZL display high sequence identity levels even in non-coding regions. This is particularly relevant since the promoter $\mathrm{CpG}$ islands of both genes remains unmethylated exclusively in germ cells, the sole cell type where they are expressed [52]. Experiments conducted in murine models have shown that Dazl expression is induced after the selective DNA demethylation of germ cell differentiation genes in post-migratory primordial germ cells, and its activation is central to the establishment of the germline genetic profile [53]. Taking into consideration both its relevance for male gametogenesis and regulation via DNA methylation, the DAZ gene family emerges as a suitable candidate for characterization of the epigenetic state of germline genes in defective spermatogenesis [54].

Based on the above hypothesis, Navarro-Costa et al., 2010 analyzed the DNA methylation pattern of the promoter $\mathrm{CpG}$ island (CGI) of two germline regulator genes-DAZL and DAZ in quality-fractioned ejaculated sperm populations from normozoospermic (NZ) and oligoasthenoteratozoospermic (OAT) men [54]. OAT patients displayed increased methylation defects in the DAZL promoter CGI when compared with NZ controls. Such differences are recorded when analyzing sperm fractions enriched either in normal or defective germ cells $(\mathrm{P}=0.001$ in both cases). Significant differences in DNA methylation profiles are also observable when comparing the qualitatively distinct germ cell fractions inside the NZ and OAT groups $(\mathrm{P}=0.003$ and $\mathrm{P}=0.007$, respectively). Contrastingly, the unmethylation pattern of the DAZ promoter CGI remains correctly established in all experimental groups. The authors concluded that incorrect epigenetic marks in germline genes may be correlated with male gametogenic defects [54].

$\mathrm{Wu}$ et al., 2010 in a study on infertile individuals analyzed the methylation pattern of DAZ (testis specific) gene in infertile individuals [55]. Methylation patterns of $\mathrm{CpG}$ island in the DAZ gene promoter region were different between somatic cells and spermatic cells in the control group. DAZ gene methylation patterns among groups with different spermatogenic status were the same in somatic cells, completely methylated. The results were concordant in spermatic cells, completely unmethylated, in groups with different degrees of spermatogenesis, except for the group with azoospermia (AZ). The authors concluded that methylation at DAZ gene promoter was not related to male infertility [55].

\section{GENETIC IMPRINTING AND MALE INFERTILITY}

Expression of alleles in a parent specific manner is named genomic imprinting. Most often this phenomenon is due to differential methylation in the $\mathrm{CpG}$ islands in one of the alleles [56]. Usually, imprinting is erased in the early germ cells $[57,58]$ and a new pattern of imprinting according to the sex of germline is established [56]. In male mice, H19 and igf2 methylation is initiated during pro-spermatogonia stage and finalized around the time of birth [59]. In human, fetal spermatogonia seem to be mostly unmethylated at H19 differentially methylated regions, although spermatogonia in adult testis demonstrate significant methylation in this region [60]. The importance of genomic imprinting during spermatogenesis for male fertility has been postulated as decreased methylation of the paternal IGF2/H19 imprinting control region 1 (ICR1) and GTL2 imprints have been found in spermatozoa of men with disturbed spermatogenesis [61]. Moreover, MEST hypermethylation was observed in men with severe oligozoospermia. A study by Marques et al. (2008) confirmed these findings by classic bisulfite sequencing of IGF2 / H19 ICR1 and MEST in spermatozoa of men with poor spermatogenesis [62]. Recently, the sixth CTCF binding site of the IGF2/H19 ICR1 has been identified as the most informative site for the association of hypomethylation and oligozoospermia and oligoasthenozoospermia [63].

Poplinski et al. (2010) determined the degree of methylation of the IGF2 / H19 imprinting control region 1 (ICR1) and MEST differentially methylated regions in swim-up purified spermatozoa from 148 idiopathic infertile men and 33 normozoospermic controls [64]. All control individuals had a high degree of IGF2/H19 ICR1 and a low degree of MEST methylation. Low sperm counts were clearly associated with IGF2 / H19 ICR1 hypomethylation and, even stronger, with MEST hypermethylation. MEST hypermethylation, but not IGF2/H19 ICR1 hypomethylation 
was found in idiopathic infertile men with progressive sperm motility below $40 \%$ and bad sperm morphology below 5\% normal spermatozoa. Ageing could be ruled out as a cause for the observed methylation defects. Sequence analysis of the CTCFL gene in peripheral blood DNA from 20 men with severe methylation defects revealed several polymorphisms, but no bona fide mutation. The authors concluded that idiopathic male infertility is strongly associated with imprinting defects at IGF2 / H19 ICR1 and MEST, with aberrant MEST methylation being a strong indicator for sperm quality. The male germ cell thus represents a potential source for aberrant epigenetic features in children conceived via ART [64].

Biossonnas et al., (2010) analyzed DNA methylation status of $47 \mathrm{CpG}$ islands located in differentially methylated regions (DMRs), the DMR0 and DMR2 of the IGF2 gene and in the 3rd and 6th CTCF-binding sites of the H19 DMR in human sperm from men with normal semen and patients with teratozoospermia (T) and/or oligoastheno-teratozoospermia (OAT) [65]. In comparison to normal level of methylation in all normal samples, reduced methylation level at variable $\mathrm{CpG}$ positions either in the IGF2 DMR2 or in both the IGF2 DMR2 and the 6th CTCF of the H19 DMR was observed in more than half of teratozoospermic patients. In the OAT group, 16 of 22 patients presented a severe loss of methylation of the 6th CTCF, closely correlated with sperm concentration. The methylation state of DMR0 and of the 3rd CTCF was never affected by the pathological status of sperm samples. The authors proposed that differential methylation at H19 locus may be a relevant marker of quantitative defects of spermatogenesis in humans [65].

\section{ASSISTED REPRODUCTION AND EPIGENETICS}

There has been remarkable progress in Assisted Reproductive Technology (ART) with the introduction of new techniques that have improved success rates. Between the years 1996 and 2007, the number of ART cycles performed in the United States more than doubled (from 64681 cycles in 1996 to 132745 in 2007) resulting in a steady increase in the number of infants born who were conceived with ART [66]. Earlier studies reported no, or small, differences in the incidence of major and minor birth defects between children conceived in vitro or in vivo [67-71]. Although the overall rate of congenital anomalies in children conceived by ART is low (4-6\%), this rate still represents a significant increase over the background rate of major malformations $(3 \%)$. Several procedures that may be used in the ART process (hormonal stimulation, egg retrieval, in vitro fertilization (IVF), intra-cytoplasmic sperm injection (ICSI), micromanipulation of gametes, exposure to culture medium, preimplantation genetic diagnosis and in vitro oocyte maturation) could subject gametes and early embryos to environmental stress [72].

Looking at the greater relative risk of low birth-weight, major and minor birth defects, and rare disorders involving imprinted genes in children conceived using in vitro techniques, Katari et al., (2009) examined DNA methylation at more than 700 genes (1536 CpG sites) in placenta and cord blood and measured gene expression levels of a subset of genes that differed in methylation levels between children conceived in vitro versus in vivo [72]. The study reported that in vitro conception is associated with lower mean methylation at $\mathrm{CpG}$ sites in placenta and higher mean methylation at $\mathrm{CpG}$ sites in cord blood. The authors also reported that in vitro conception-associated DNA methylation differences are associated with gene expression differences at both imprinted and non-imprinted genes. The range of inter-individual variation in gene expression of the in vitro and in vivo groups overlaps substantially but some individuals from the in vitro group differ from the in vivo group mean by more than two standard deviations. Several of the genes whose expression differs between the two groups have been implicated in chronic metabolic disorders, such as obesity and type II diabetes. These findings suggest that there may be epigenetic differences in the gametes or early embryos derived from couples undergoing treatment for infertility, which may affect long term pattern of gene expression and disease susceptibility [72].

\section{CONCLUSION AND FUTURE DIRECTIONS}

Changes in epigenenome are now well known to affect gene expression, and several genes participating in spermatogenesis are epigenetically regulated. Differential methylation in promoter regions of certain genes contributes to male infertility, while epigenetic changes in others do not. Epigenetic modifications not only affect spermatogenesis but also affect disease susceptibility later in life. Endocrine disruptors known to adversely affect spermatogenesis could operate by affecting epigenetic modifications. Epigenetic modifications thus incurred not only affect short term gene expression in the generation affected, but also affect long term gene expression and the effect could be visible in the coming generations. This could result in imprinting of certain genes which affect phenotype and are well inherited. Changes due to epigenome could differ between children born by assisted reproduction techniques and natural conception. Preliminary studies in this field indicate possible differences, however, more numbers of studies with long term follow up of the children thus born could highlight any changes attributable to epigenetic changes due to in vitro methods.

Overall, not many genes important for spermatogenesis have been analyzed for epigenetic modifications in male infertility. Therefore, the field is rather young and more investigations to explore epigenetic modifications in genes regulating spermatogenesis are required. We have much to learn about the role of epigenetics in spermatogenesis and male infertility. Particular emphasis should be given to the genes having $\mathrm{CpG}$ islands in the promoter regions. Epigenetics bears the promise of reversing the effect due to its dynamic nature. It is possible that deep understanding of the epigenetic processes and their influence could show us the path to therapies based on epigenome modifications. This has an edge over genetic studies since we can offer cure to the individuals bearing epigenetic changes in contrast to individuals having genetic modifications.

\section{EXPERT COMMENTARY}

Newer research everyday is pointing out the important of epigenetic modifications. Epigenetic modifications are now 
known to affect gene expression and susceptibility to almost every disease. Several genes participating in spermatogenesis are epigenetically regulated. Accordingly aberrant epigenetic modifications at several loci are now known to contribute to abnormalities in spermatogenesis possibly leading to male infertility. Differentially methylated regions, abnormal chromatin remodelling and imprinting defects could all contribute to male infertility. It has also been proposed that differences in epigenetic modifications could account for health problems encountered in the individuals born using in vitro techniques. However, the field is yet in its infancy and further research may identify several other regions which are epigenetically regulated, defects at which could contribute to male infertility or differences in the individuals born by natural conception and assisted reproduction. Several regions of genome could be imprinted to regulate spermatogenesis and fertility. The field is open to further research to decode more codes of the epigenome.

\section{FIVE YEAR REVIEW}

Though the knowledge of $\mathrm{CpG}$ islands is an old phenomenon, the dynamics of these islands have been explored only in the last decade. Research in the recent years has revealed how $\mathrm{CpG}$ islands could regulate gene expression and the development of a particular phenotype. Apart from methylation, other epigenetic modifications such as chromatin remodelling and genetic imprinting are rather new concepts and have gained attention only recently. Genetic imprinting now appears to be an important concept which could fill the gaps in the knowledge of spermatogenesis regulation and development of infertility phenotypes. Several loci are known to be imprinted but the number of loci unknown at present could outnumber those known to be imprinted. Several environmental hazards could affect spermatogenesis and male fertility by epigenetic modifications. Vinclozolin is a very well explored environmental hazard affecting epigenetic modifications; however, more such substances particularly endocrine disruptors need to be evaluated for their effects on epigenome. There has been only little research on other such substances which now deserve attention.

\section{KEY POINTS}

- Several genes participating in spermatogenesis are epigenetically regulated.

- Modifications in differentially methylated regions could contribute to male infertility.

- Genetic imprinting could account for regulation of spermatogenesis and development of a particular infertility phenotype.

- Aberrant epigenetic modifications in male infertility include changes in methylation, chromatin remodelling and aberrant imprinting.

- $\quad$ Environmental hazards such as endocrine disruptors could affect fertility by catering modifications in the epigenome.
- In vitro procedures for fertility treatment could change epigenome, accounting for health implications in the individuals born using these methods.

\section{ACKNOWLEDGEMENTS}

None Declared.

\section{CONFLICT OF INTEREST}

None Declared.

\section{REFERENCES}

[1] Gianotten J, Lombardi MP, Zwinderman AH, Lilford RJ, van der Veen F. Idiopathic impaired spermatogenesis: Genetic epidemiology is unlikely to provide a short-cut to better understanding. Hum Reprod Update 2004; 10: 533-9.

[2] Anway MD, Cupp AS, Uzumcu M, Skinner MK. Epigenetic transgenerational actions of endocrine disruptors and male fertility. Science 2005; 308: 1466-9.

[3] Li ZX, Ma X, Wang ZH. A differentially methylated region of the DAZ1 gene in spermatic and somatic cells. Asian J Androl 2006; 8: 61-7.

[4] Egger G, Liang G, Aparicio A, Jones PA. Epigenetics in human disease and prospects for epigenetic therapy. Nature 2004; 429 : 457-63.

[5] Takai D, Jones PA. Comprehensive analysis of $\mathrm{CpG}$ islands in human chromosomes 21 and 22. Proc Natl Acad Sci USA 2002; 99: 3740-5.

[6] Bird A, Tate P, Nan X, et al. Studies of DNA methylation in animals. J Cell Sci 1995; 19(Suppl): 37-9.

[7] Biermann K, Steger K. Epigenetics in male germ cells. J Androl 2007; 28: 466-80.

[8] Zhang X, Yazaki J, Sundaresan A, et al. Genome-wide highresolution mapping and functional analysis of DNA methylation in Arabidopsis. Cell 2006; 126: 1189-201.

[9] Gardiner-Garden M, Frommer M. CpG islands in vertebrate genomes. J Mol Biol 1987; 2: 261-82.

[10] Okano M, Xie S, Li E. Cloning and characterization of a family of novel mammalian DNA (cytosine-5) methyltransferases. Nat Genet 1998; 19: 219-20.

[11] Bestor TH. The DNA methyltransferases of mammals. Hum Mol Genet 2000; 9: 2395-402.

[12] Reik W, Dean W, Walter J. Epigenetic reprogramming in mammalian development. Science 2001; 293: 1089-93.

[13] Li E. Chromatin modification and epigenetic reprogramming in mammalian development. Nat Rev Genet 2002; 3: 662-73.

[14] Leonhardt H, Page AW, Weier HU, Bestor TH. A targeting sequence directs DNA methyltransferase to sites of DNA replication in mammalian nuclei. Cell 1992; 71: 865-73.

[15] Liu Y, Oakeley EJ, Sun L, Jost JP. Multiple domains are involved in the targeting of the mouse DNA methyltransferase to the DNA replication foci. Nucleic Acids Res 1998; 26: 1038-45.

[16] Ooi SK, Bestor TH. The colorful history of active DNA demethylation. Cell 2008; 133: 1145-8.

[17] Fischle W, Wang Y, Jacobs SA, Kim Y, Allis CD, Khorasanizadeh S. Molecular basis for the discrimination of repressive methyllysine marks in histone H3 by polycomb and HP1 chromodomains. Genes Dev 2003; 17: 1870-81.

[18] Noma K, Allis CD, Grewal SI. Transitions in distinct histone H3 methylation patterns at the heterochromatin domain boundaries. Science 2001; 293: 1150-5.

[19] Santos-Rosa H, Schneider R, Bannister AJ, et al. Active genes are tri-methylated at K4 of histone H3. Nature; 419: 407-11.

[20] Berger SL. Histone modifications in transcriptional regulation. Curr Opin Genet Dev 2002; 12: 142-8.

[21] Bottomley MJ. Structures of protein domains that create or recognize histone modifications. EMBO Reports 2004; 5: 464-9. 
[22] Fernandez-Capetillo O, Mahadevaiah SK, Celeste A, et al. H2AX is required for chromatin remodeling and inactivation of sex chromosomes in male mouse meiosis. Dev Cell 2003; 4: 497-508.

[23] Baarends WM, Wassenaar E, Hoogerbrugge JW, et al. Loss of HR6B ubiquitin-conjugating activity results in damaged synaptonemal complex structure and increased crossing-over frequency during the male meiotic prophase. Mol Cell Biol 2003; 23: 1151-62.

[24] Zhu B, Zheng Y, Pham AD, et al. Monoubiquitination of human histone H2B: The factors involved and their roles in HOX gene regulation. Mol Cell 2005; 20: 601-11.

[25] Shiio Y, Eisenman RN. Histone sumoylation is associated with transcriptional repression. Proc Natl Acad Sci USA 2003; 100: 13225-30.

[26] Narlikar GJ, Fan HY, Kingston RE. Cooperation between complexes that regulate chromatin structure and transcription. Cell 2002; 108: 475-87.

[27] Partensky PD, Narlikar GJ. Chromatin remodelers act globally, sequence positions nucleosomes locally. J Mol Biol 2009; 391: 1225.

[28] Fan HY, He X, Kingston RE, Narlikar GJ. Distinct strategies to make nucleosomal DNA accessible. Mol Cell 2003; 11: 1311-22.

[29] Fan HY, Trotter KW, Archer TK, Kingston RE. Swapping function of two chromatin remodeling complexes. Mol Cell 2005; 17: 80515.

[30] Yates JA, Menon T, Thompson BA, Bochar DA. Regulation of HOXA2 gene expression by the ATP-dependent chromatin remodeling enzyme CHD8. FEBS Lett 2010; 584: 689-93.

[31] Skinner MK, Manikkam M, Guerrero-Bosagna C. Epigenetic transgenerational actions of environmental factors in disease etiology. Trends Endo Met 2010; 21: 214-22.

[32] Jirtle RL, Skinner MK. Environmental epigenomics and disease susceptibility. Nat Rev Genet 2007; 8: 253-62.

[33] Anway MD, Cupp AS, Uzumcu M, Skinner MK. Epigenetic transgenerational actions of endocrine disruptors and male fertility. Science 2005; 308: 1466-9.

[34] Wong C, Kelce WR, Sar M, Wilson EM. Androgen receptor antagonist versus agonist activities of the fungicide vinclozolin relative to hydroxyflutamide. J Biol Chem 1995; 270: 19998-20003.

[35] Anway MD, Memon MA, Uzumcu M, Skinner MK. Transgenerational effect of the endocrine disruptor vinclozolin on male spermatogenesis. J Androl 2006; 27: 868-79.

[36] Anway MD, Leathers C, Skinner MK. Endocrine disruptor vinclozolin induced epigenetic transgenerational adult-onset disease. Endocrinology 2006; 147: 5515-23.

[37] Popp C, Dean W, Feng S, et al. Genome-wide erasure of DNA methylation in mouse primordial germ cells is affected by AID deficiency. Nature 2010; 463: 1101-5.

[38] Guerrero-Bosagna C, Settles M, Lucker B, Skinner MK. Epigenetic transgenerational actions of vinclozolin on promoter regions of the sperm epigenome. PLoS One 2010; 5: 13100.

[39] Steilmann C, Cavalcanti MC, Bartkuhn M, et al. The interaction of modified histones with the bromodomain testis-specific (BRDT) gene and its mRNA level in sperm of fertile donors and subfertile men. Reproduction 2010; 140: 435-43.

[40] Kimmins S, Sassone-Corsi P. Chromatin remodeling and epigenetic features of germ cells. Nature 2005; 434: 583-89.

[41] Davie JR. Covalent modifications of histones: expression from chromatin templates. Curr Opin Genet Dev 1998; 8: 173-8.

[42] Rousseaux S, Reynoird N, Escoffier E, Thevenon J, Caron C, Khochbin S. Epigenetic reprogramming of the male genome during gametogenesis and in the zygote. Reprod Biomed Online 2008; 16: 492-503.

[43] Steilmann C, Cavalcanti MC, Bergmann M, Kliesch S, Weidner W, Steger K. Aberrant mRNA expression of chromatin remodelling factors in round spermatid maturation arrest compared with normal human spermatogenesis. Mol Hum Reprod 2010; 16: 726-33.

[44] Chen Z, Karaplis AC, Ackerman SL, et al. Mice deficient in methylenetetrahydrofolate reductase exhibit hyperhomocysteinemia and decreased methylation capacity, with neuropathology and aortic lipid deposition. Hum Mol Genet 2001; 10: 433-43.

[45] Leopardi P, Marcon F, Caiola S, et al. Effects of folic acid deficiency and MTHFR C677T polymorphism on spontaneous and radiation-induced micronuclei in human lymphocytes. Mutagenesis 2006; 21: 327-33.

[46] Marques CJ, Costa P, Vaz B, et al. Abnormal methylation of imprinted genes in human sperm is associated with oligozoospermia. Mol Hum Reprod 2008; 14: 67-74.

[47] Khazamipour N, Noruzinia M, Fatehmanesh P, Keyhanee M, Pujol P. MTHFR promoter hypermethylation in testicular biopsies of patients with non-obstructive azoospermia: the role of epigenetics in male infertility. Hum Reprod 2009; 24: 2361-4.

[48] Hammoud SS, Purwar J, Pflueger C, Cairns BR, Carrell DT. Alterations in sperm DNA methylation patterns at imprinted loci in two classes of infertility. Fertil Steril 2010; 94: 1728-33.

[49] Liu Z, Zhou S, Liao L, Chen X, Meistrich M, Xu J. Jmjd1a demethylase-regulated histone modification is essential for cAMPresponse element modulator-regulated gene expression and spermatogenesis. J Biol Chem 2010; 285: 2758-70.

[50] Navarro-Costa P, Goncalves J, Plancha CE. The AZFc region of the $Y$ chromosome: at the crossroads between genetic diversity and male infertility. Hum Reprod Update 2010; 16: 525-42.

[51] Lin Y, Page DC. Dazl deficiency leads to embryonic arrest of germ cell development in XY C57BL/6 mice. Dev Biol 2005; 288: 30916.

[52] Yen PH. Putative biological functions of the DAZ family. Int J Androl 2004; 27: 125-9.

[53] Haston KM, Tung JY, Reijo Pera RA. Dazl functions in maintenance of pluripotency and genetic and epigenetic programs of differentiation in mouse primordial germ cells in vivo and in vitro. PLoS ONE 2009; 4: 5654.

[54] Navarro-Costa P, Nogueira P, Carvalho M, et al. Incorrect DNA methylation of the DAZL promoter $\mathrm{CpG}$ island associates with defective human sperm. Hum Reprod 2010; 25: 2647-54.

[55] Wu W, Lu C, Xia Y, et al. Lack of association between DAZ gene methylation patterns and spermatogenic failure. Clin Chem Lab Med 2010; 48: 355- 60 .

[56] Reik W, Dean W, Walter J. Epigenetic reprogramming in mammalian development. Science 2001; 293: 1089- 92.

[57] Brandeis M, Ariel M, Cedar H. Dynamics of DNA methylation during development. Bioessays 1993; 11: 709- 13.

[58] Constancia M, Pickard B, Kelsey G, Reik W. Imprinting mechanisms. Genome Res 1998; 8: 881-900.

[59] La Salle S, Mertineit C, Taketo T, et al. Windows for sex-specific methylation marked by DNA methyltransferase expression profiles in mouse germ cells. Dev Biol 2004; 268: 403-15.

[60] Kerjean A, Dupont JM, Vasseur C, et al. Establishment of the paternal methylation imprint of the human H19 and MEST/PEG1 genes during spermatogenesis. Hum Mol Genet 2000; 9: 2183-7.

[61] Kobayashi H, Sato A, Otsu E, et al. Aberrant DNA methylation of imprinted loci in sperm from oligospermic patients. Hum Mol Genet 2007; 16: 2542-51.

[62] Marques CJ, Costa P, Vaz B, et al. Abnormal methylation of imprinted genes in human sperm is associated with oligozoospermia. Mol Hum Reprod 2008; 14: 67-74.

[63] Boissonnas CC, Abdalaoui HE, Haelewyn V, et al. Specific epigenetic alterations of IGF2-H19 locus in spermatozoa from infertile men. Eur J Hum Genet 2010; 18: 73-80.

[64] Poplinski A, Tüttelmann F, Kanber D, Horsthemke B, Gromoll J. Idiopathic male infertility is strongly associated with aberrant methylation of MEST and IGF2/H19 ICR1. Int J Androl 2010; 33: $642-9$.

[65] Boissonnas CC, Abdalaoui HE, Haelewyn V, et al. Specific epigenetic alterations of IGF2-H19 locus in spermatozoa from infertile men. Eur J Hum Genet 2010; 18: 73-80.

[66] Society for Assisted Reproductive Technology (2008) National data Summary Retrieved from https://www.sartcorsonlinecom/ rptCSR_PublicMultYearaspx?ClinicPKID

[67] Ericson A, Kallen B. Congenital malformations in infants born after IVF: a population-based study. Hum Reprod 2001; 16: 504-9.

[68] Koivurova S, Hartikainen AL, Gissler M, Hemminki E, Sovio U, Jarvelin MR. Neonatal outcome and congenital malformations in children born after in-vitro fertilization. Hum Reprod 2002; 17 : 1391-8.

[69] Hansen M, Kurinczuk JJ, Bower C, Webb S. The risk of major birth defects after Intracytoplasmic sperm Injection and in vitro fertilization. NEJM 2002; 346: 725-30. 
[70] Hansen M, Bower C, Milne E, de Klerk N, Kurinczuk J. Assisted reproductive technologies and the risk of birth defects-a systematic review. Hum Reprod 2005; 20: 328-38.

[71] Reefhuis J, Honein MA, Schieve LA, Correa A, Hobbs CA, Rasmussen SA. Assisted reproductive technology and major structural birth defects in the United States. Hum Reprod 2008; 24: 360- 6 .

[72] Katari S, Turan N, Bibikova M, et al. DNA methylation and gene expression differences in children conceived in vitro or in vivo. Hum Mol Genet 2009; 18: 3769-78.

Received: January 02, 2010

Revised: March 08, 2010

Accepted: July 26, 2010

(C) Rajender and Agarwal; Licensee Bentham Open.

This is an open access article licensed under the terms of the Creative Commons Attribution Non-Commercial License (http://creativecommons.org/licenses/bync/3.0/), which permits unrestricted, non-commercial use, distribution and reproduction in any medium, provided the work is properly cited. 